\title{
Steel Beam-Column Joint with Discontinuous Vertical Reinforcing Bars
}

\author{
Ju-Yun $\mathrm{HU}^{1}$, Won-Kee HONG ${ }^{1}{ }^{*}$, Seon-Chee PARK ${ }^{1}$, Jisoon $_{\mathrm{KIM}}{ }^{1}$ \\ ${ }^{1}$ Department of Architectural Engineering, Kyung Hee University, \\ Yongin 446-701, Republic of Korea \\ *Corresponding author's e-mail: hongwk@khu.ac.kr
}

\begin{abstract}
The authors proposed steel beam-column connections for precast concrete frames in previous studies. The steel-concrete composite frames provided fast assembly time as steels with economy of concrete structures. However, when enough space is not available at column-beam joints steel sections from beams cannot be connected with column brackets. This paper suggests that some vertical reinforcing bars are disconnected at joints by connecting vertical steel reinforcements to steel plates placed above and below column steels to provide load transferring path. Loads from re-bars are transferred to steel plates, column steels and back to steel plates and re-bars below column steels. Re-bars connected to steel plates by bolts at above and below column steel are discontinued at joint to provide spaces for connections between column brackets and beam steels. Extensive experiments were performed to verify load transfer from re-bars to steel plates above joints and steel plates to re-bars below joint. The flexural load bearing capacity of a column with total of 24 vertical re-bars were compared to columns with discontinuous re-bars at joints. The number of discontinuous re-bars at joint used in column specimen was $0(0.0 \%), 4(16.7 \%), 12$ $(50.0 \%)$, and $20(83.3 \%)$. The numbers in parenthesis are the percentages of discontinuous rebars to the total number of vertical re-bars of control column. Experiments showed how loads from vertical steel reinforcements that were cut off at joints were transferred to steel plate. The test results also demonstrated that a part of flexural capacities were reduced for specimen with discontinuous vertical re-bars. The reduction of $6.0 \%, 13.7 \%$ and $54.0 \%$ of flexural capacities were observed for columns with 4 (16.7\%), 12 (50.0\%) and $20(83.3 \%)$ discontinuous vertical rebars, respectively. The test results can be used to design vertical reinforcing bars and column joints that can provide space for column brackets to which steel members of beams are connected.
\end{abstract}

\section{KEYWORDS}

steel beam-column joint; load path; beam-column connection; flexural capacity; steel-concrete composite frame; discontinuous re-bar, inelastic finite element analysis

\section{INTRODUCTION}

In case where there were structural details conflicting between vertical column re-bars and column brackets during the construction of steel-concrete composite frames, spaces should be provided enough to place these structural components where they should be. This conflict as shown in Figure 1 can be resolved by disconnecting vertical re-bars at the sacrifice of some reductions in moment resisting capacity. This strength decrease can be, however, minimized or 
compensated by introducing high strength steel plate at joints. New beam-column joint connections for steel-concrete composite frames were suggested with structural details based on both extensive experimental and analytical investigations.

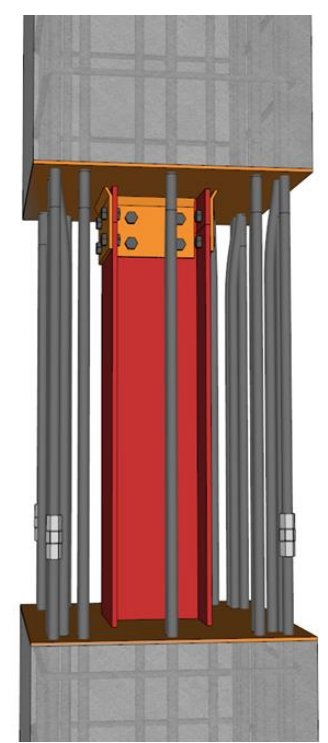

Figure 1. Joint details conflicting between vertical column re-bars and column bracket

\section{METHOD OF EXPERIMENTAL INVESTIGATION}

Figure 2(a) illustrates test specimen with 4 vertical reinforcing bars being continuous while 20 vertical re-bars were cut off by connecting them to steel plates by bolts at above and below column steel. Specimens were designed to verify load path along vertical re-bars. Steel plate above column steel picked loads from re-bars as shown in Figure 2(b). The loads moved down to steel plate through column steel and further down to lower steel plate. Finally column loads were picked up by vertical re-bars again. The load capacity lost during the path was measured by experiment and recommendations were made for joint connection design. The flexural load bearing capacity of a control column with a total of 24 vertical re-bars were compared to that of columns which lost 0, 4 (16.7\%), 12 (50.0\%), and 20 (83.3\%) re-bars, respectively. The numbers in parenthesis are the percentages of discontinuous re-bars to the total number of vertical re-bars of control column. Figure 2(c) shows the structural details of bolted connection between re-bars and plate. Figure 3(a) shows test set up for the specimens with details for vertical reinforcing bars bolted to plates. The distance from the base to the centre of loading was set as $1.5 \mathrm{~m}$. The test was loaded by displacement control method with the loading protocol as shown in Figure 3(b). The actuator of $1000 \mathrm{kN}$ with stroke of $300 \mathrm{~mm}$ was used to exert cyclic loading to the specimens. LVDTs with vertical and horizontal direction were installed as shown in Figure 3(a) to accurately measure deformation of specimens. Figure 4 shows strain gauges to measure strains of re-bars at various locations. Figure 5 depicts the deformed specimen under load application. 


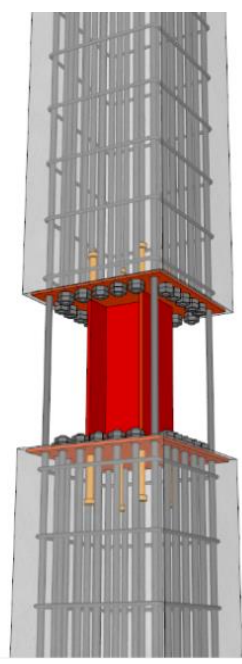

Figure 2 (a). Column Re-bar Discontinuity

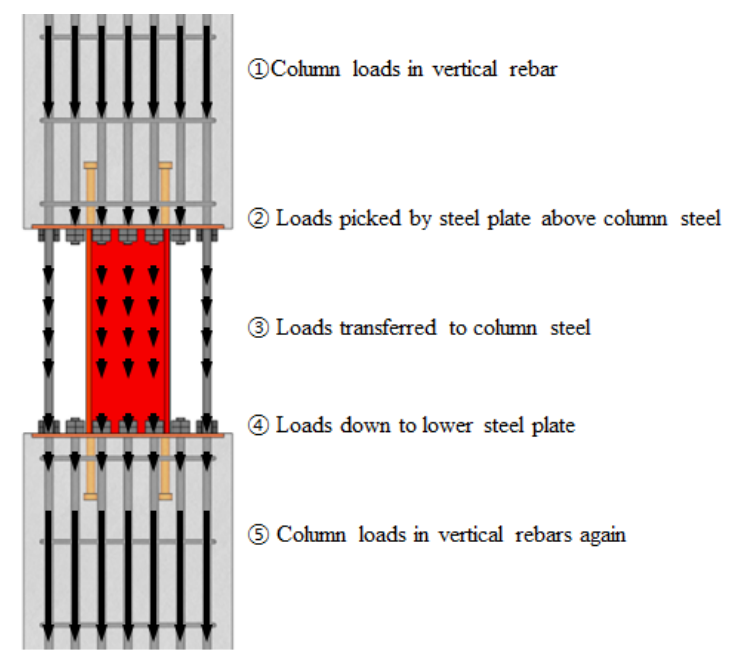

Figure 2 (b). Load path along with Column

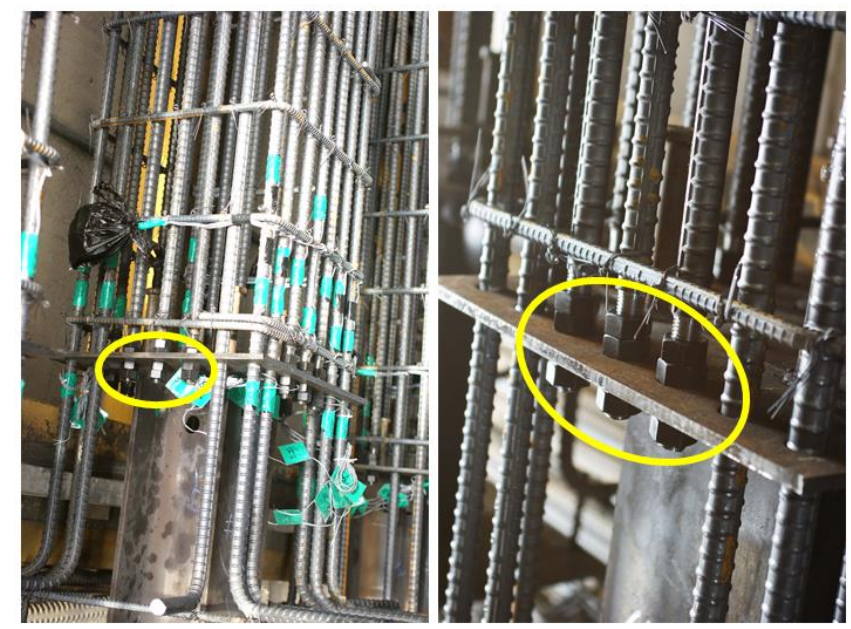

Figure 2 (c). Discontinuous re-bars connected to steel plates by bolts (Specimen \#3)

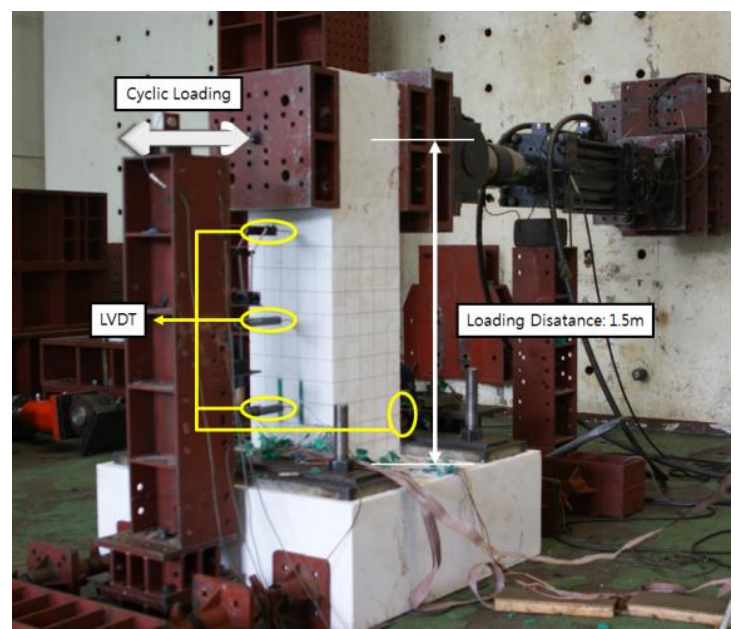

Figure 3 (a). Test set up for the specimens

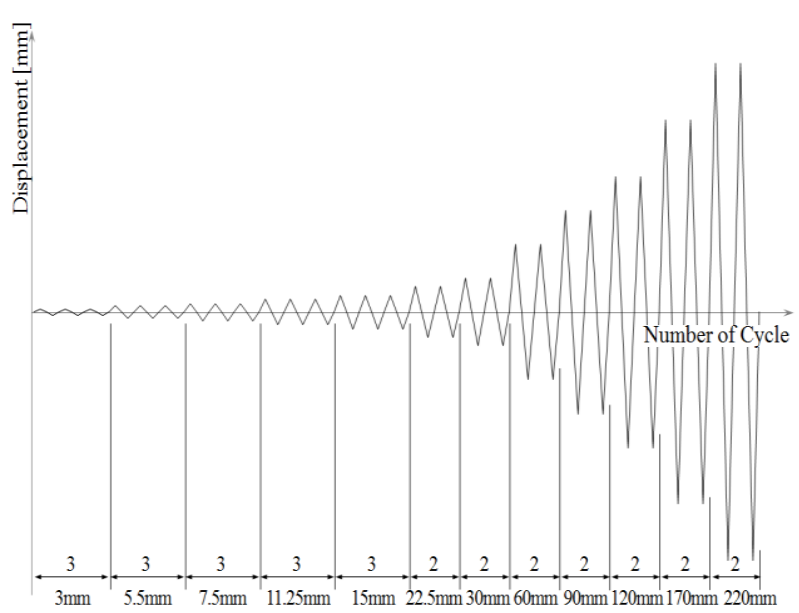

Figure 3 (b). Loading protocol for loading 


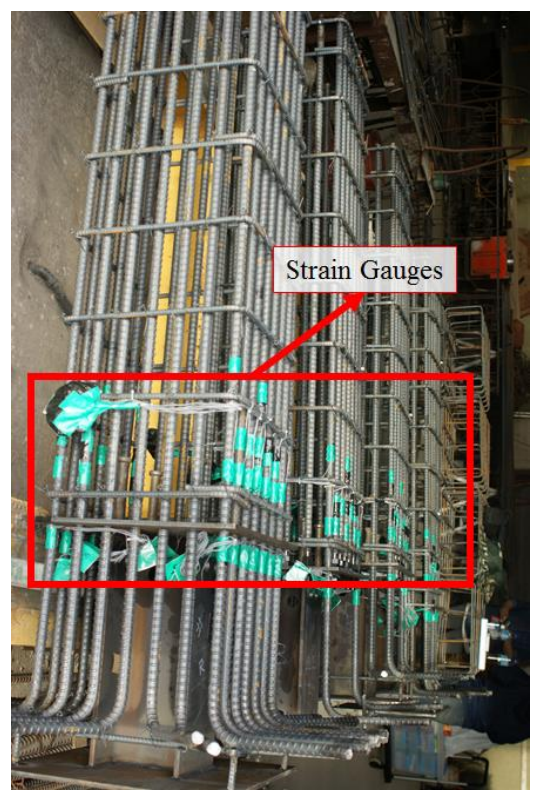

Figure 4. Strain gauges of re-bars

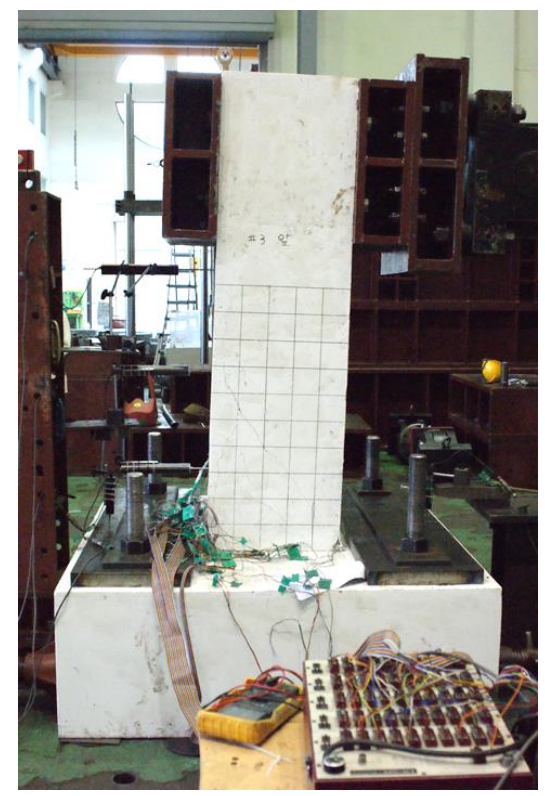

Figure 5. The deformed specimen under load application

\section{PREPARATION OF TEST SPECIMENS}

Figure 6 illustrates four specimens tested to verify load transfer from re-bars to steel plates above joints and steel plates to re-bars below joint. The flexural load bearing capacity of a column with total of 24 vertical re-bars were compared to that of columns with discontinuous re-bars at joints. The number of discontinuous re-bars at joint used in column specimens was $0(0.0 \%$, Specimen \#1), 4 (16.7\%, Specimen \#2), 12 (50.0\%, Specimen \#3), and 20 (83.3\%, Specimen \#4) as depicted in Figure 6. The material properties of the specimens are listed in Table 1.

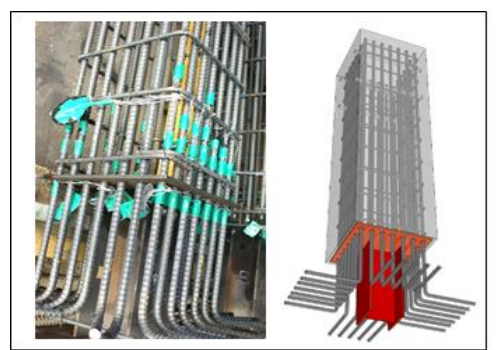

Specimen \#1

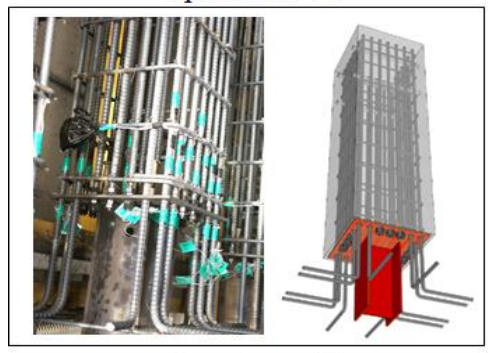

Specimen \#3

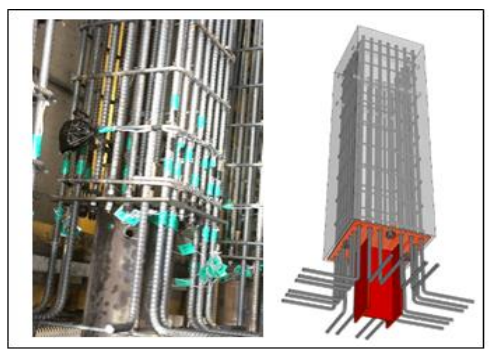

Specimen \#2

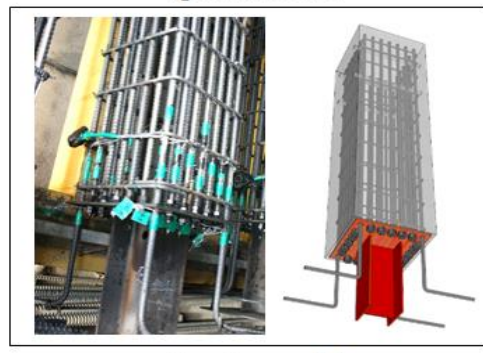

Specimen \#4

Figure 6. Specimens \#1, \#2, \#3 and \#4 
Table 1. Material properties used in the specimen

\begin{tabular}{|c|c|}
\hline \multicolumn{2}{|c|}{ Material Property } \\
\hline Concrete Compressive Strength $\left(f_{c}^{\prime}\right)$ & $27 \mathrm{MPa}$ \\
\hline Rebar Yield Strength $\left(f_{y}\right)$ & $400 \mathrm{MPa}$ \\
\hline Steel Yield Strength $\left(F_{y}\right)$ & $325 \mathrm{MPa}$ \\
\hline
\end{tabular}

\section{Experiment and Analytical Results}

Figures 7, 8, 9 and 10 demonstrate the hysteretic load-displacement relationships of Specimen $\# 1$, \#2, \#3 and \#4, respectively. The displacements were related with strains through gauge readings plotted on the load-displacement curves. These displacements were also related to the analytical data calculated based on strain compatibility as shown in Figure 10. The comparison between analytical and experimental load-displacement relationship in Figure 10 indicated that discontinued 20 re-bars contributed to moment resisting capacity. All of the specimens demonstrated good ductility, showing well established load path. Table 2 presents the measured maximum load resisting capacity of each specimen. As shown in Table 2, the maximum load resisting capacity decreased as the number of discontinuous reinforcing bars increased at joint. Specimen \#1 which was the control specimen with no re-bar discontinuities was compared with the remaining Specimens (\#2, \#3 and \#4) to observe the reduction of moment resisting capacity for these specimens. Moment resisting capacity of $6 \%$ was reduced for Specimen $\# 2$ with 4 rebar discontinuities while the reduction of moment resisting capacity of the $54.1 \%$ was observed for the Specimen \#4 which lost 20 re-bars at column joint. The capacity drop for Specimen \#3 with $50 \%$ of re-bar discontinuities was found to be only $13.7 \%$ compared with that of control specimen, indicating load transfer was relatively well established between upper re-bar, upper plate, steel section at joint, lower plate, and to lower re-bar as shown Figure 2 (b).

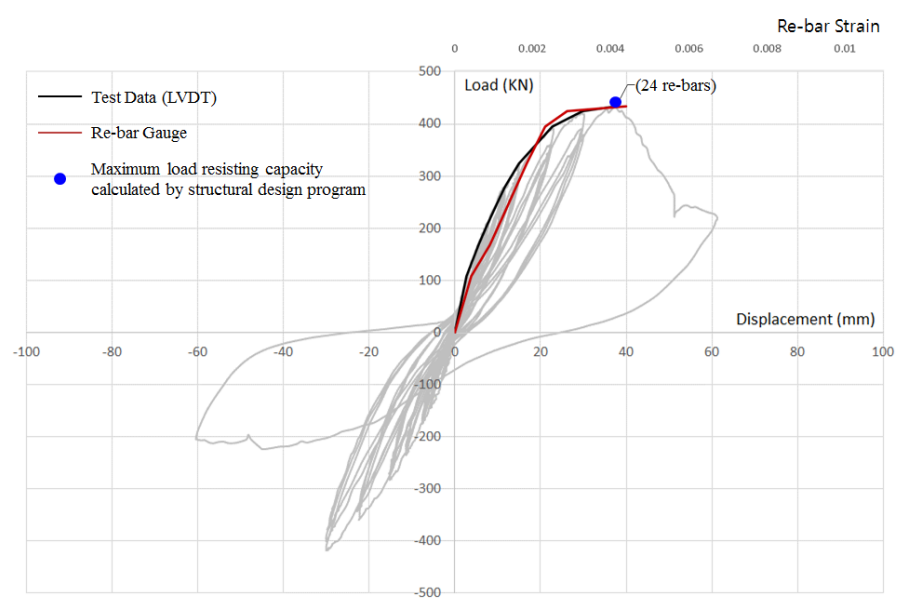

Figure 7. Load-displacement curve of Specimen \#1 


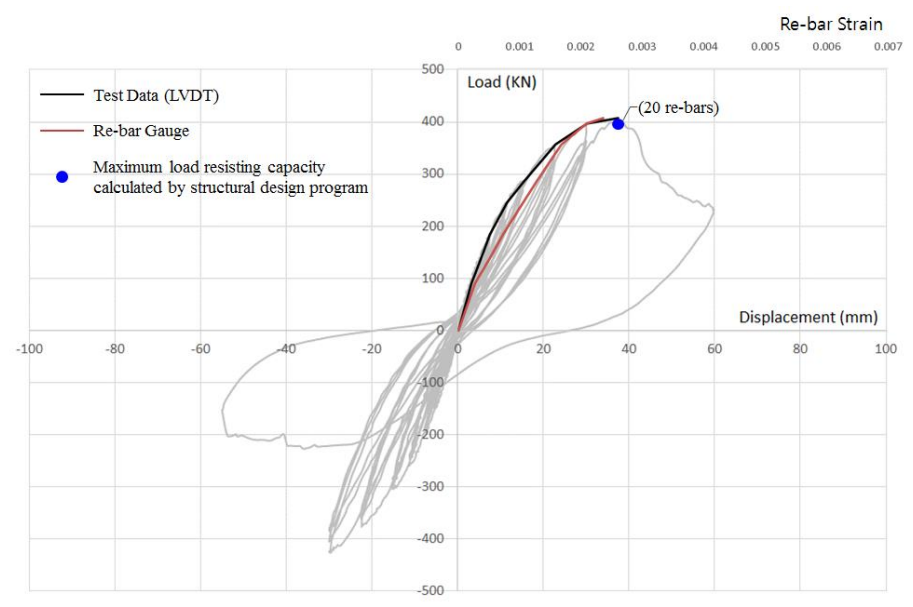

Figure 8. Load-displacement curve of Specimen \#2

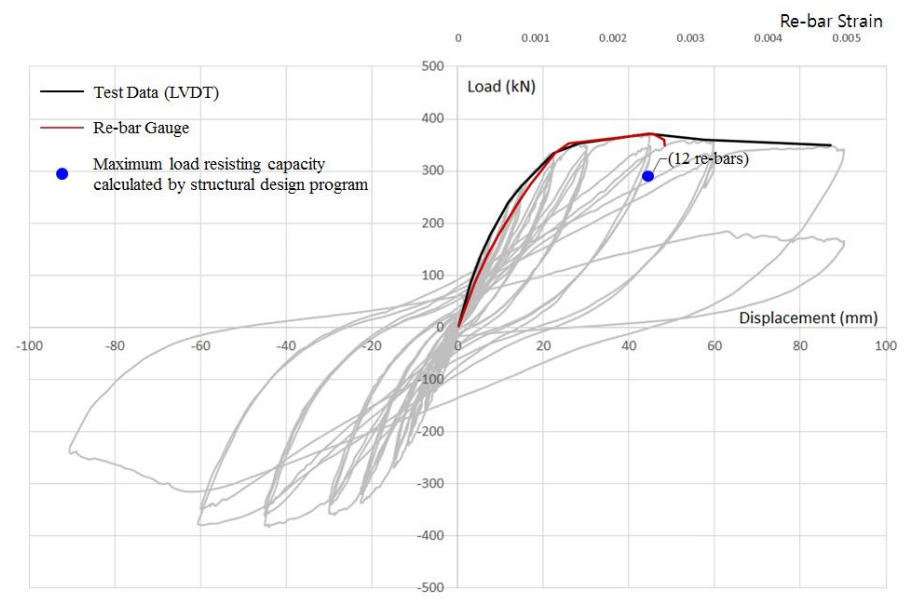

Figure 9. Load-displacement curve of Specimen \#3

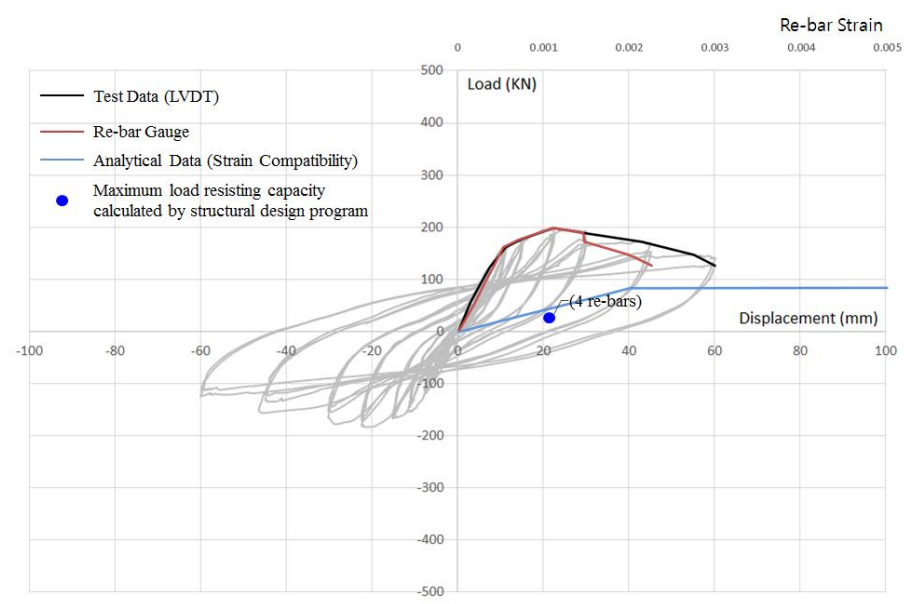

Figure 10. Load-displacement curve of Specimen \#4 
Table 2. Number of discontinuous re-bars and Moment resisting capacity of the Specimen \#1, \#2, \#3 and \#4

\begin{tabular}{|c|c|c|c|c|}
\cline { 2 - 5 } \multicolumn{1}{c|}{} & Specimen \#1 & Specimen \#2 & Specimen \#3 & Specimen \#4 \\
\hline $\begin{array}{c}\text { Discontinuous re-bar ratio } \\
\text { (Number of re-bars) }\end{array}$ & $0.0 \%$ & $16.7 \%$ & $50.0 \%$ & $83.3 \%$ \\
(0 re-bar cut off) & (4 re-bars cut off) & (12 re-bars cut off) & (20 re-bars cut off) \\
\hline Reduction Ratio & $0.0 \%$ & $6.0 \%$ & $13.7 \%$ & $54.0 \%$ \\
(Moment resisting capacity) & $(646.80 \mathrm{kN}-\mathrm{m})$ & $(608.13 \mathrm{kN}-\mathrm{m})$ & $(557.10 \mathrm{kN}-\mathrm{m})$ & $(297.48 \mathrm{kN}-\mathrm{m})$ \\
\hline
\end{tabular}

Figure 11 presents the degradation tendency of flexural moment resisting capability of columns vs. columns with re-bar discontinuity. The strength decreased to 94.0\%(Specimen \#2), $86.3 \%$ (Specimen \#3), and down to $46.0 \%$ (Specimen \#4) as re-bar discontinuity increased to 16.7\%(Specimen \#2), 50.0\%(Specimen \#3), 83.3\%(Specimen \#4), respectively.

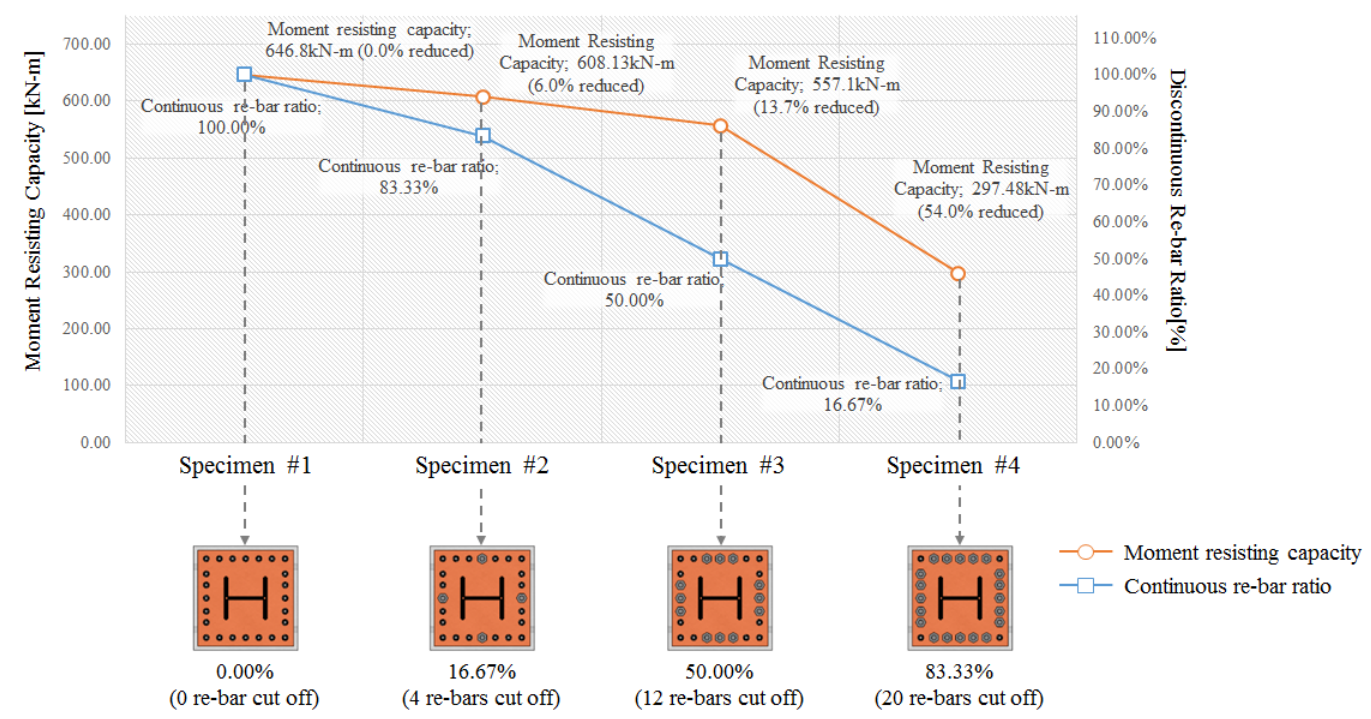

Figure 11. Comparison between number of continuous reinforcing bars vs. maximum moment resisting capacity

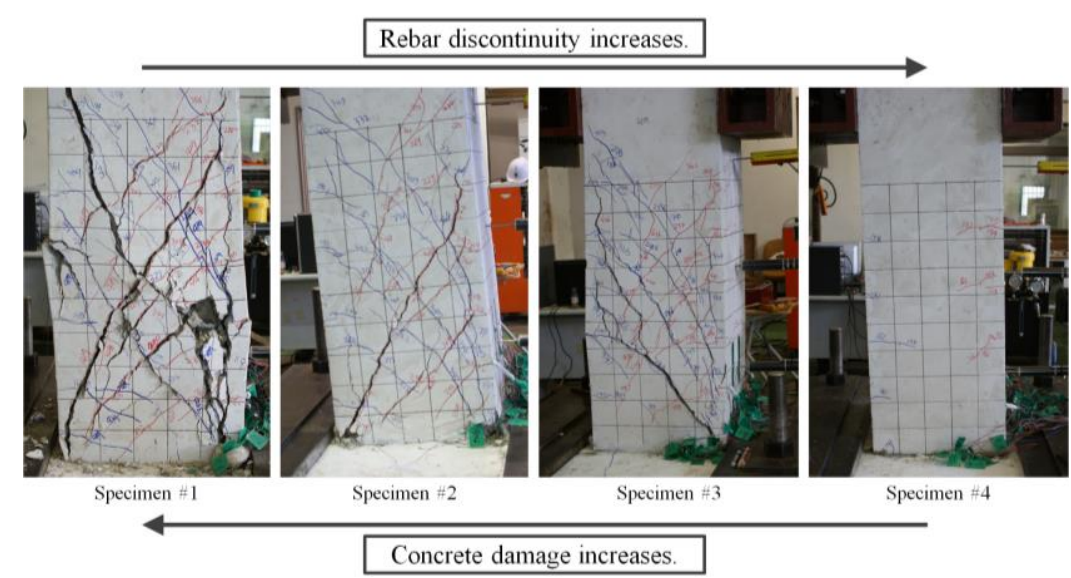

Figure 12. Degree of deterioration of specimens 
Figure 12 presents degree of deterioration of specimens which were different from each other, depending on which structural components were damaged. Concrete was most severely damaged for Specimen \#1 while Specimen \#4 showed least damage on the face of concrete. As can be seen in this figure, more concrete deteriorations were observed as less re-bars were discontinued. Least concrete deterioration occurred to Specimen \#4 since most structural damages were concentrated with re-bars with bolts connection, preventing concrete from suffering severe structural deterioration. The inelastic finite element analysis is in progress to identify additional factors that influenced the degradation of structural capacity during test.

\section{CONCLUSIONS}

This paper recognized there were some structural details conflicting between vertical column rebars and column brackets, limiting spaces not enough to place these structural components where they should be. This paper delved a solution to provide structural details that can be constructible at joints by disconnecting vertical re-bars. In this paper, spaces for connections at beam-column joint for steel-concrete composite frames were provided at the sacrifice of some reductions in moment resisting capacity. This strength decrease can be, however, minimized or compensated by introducing high strength steel plate at joints. The loading path was found to be well established in the specimens, allowing load transfer between upper re-bar, upper plate, steel section at joint, lower plate, and to lower re-bar. Experimental investigation demonstrated that load resisting capacities were decreasing fast as more re-bars were disconnected at joints. Only $6 \%$ reduction in load resisting capacity was observed for the specimen with $17 \%$ re-bar cut off while $54 \%$ strength reduction were found for the specimen with $83 \%$ re-bar cut off. The findings in this paper are expected to be of valuable data for the research for replacing the conventional beamcolumn joint by extended beam end plate. A research for the extended beam end plate with high yield strength is anticipated in the future to restore the load resisting capability which was lost.

\section{ACKNOWLEDGEMENTS}

This work was supported by the Technology Transfer Center for National R\&D Program(TTC) grant funded by the Korea government(MSIP)(No. 2014K000239).

\section{REFERENCES}

AISC (2011). ASCI Steel Construction Manual 14th, ANSI/AISC 360-10 Specification for Structural Steel Buildings, Chapter I Design of Composite Members, Chicago. American Institute of Steel Construction (AISC). (2011)

AISC (2011). AISC Examples Version 14.0, Chicago. American Institute of Steel Construction (AISC). (2011).

Hong, W.K., Kim, J.M., Park, S.C., Kim, S.I., Lee, S.G., Lee, H.C. and Yoon, K.J. (2009). "Composite Beam Composed of Steel and Pre-cast Concrete. (Modularized Hybrid System, MHS) Part II: Analytical Investigation”, Structural Design of Tall and Special Buildings, 18(12), 891-905. 